\title{
Inverse modelling-based reconstruction of the Chernobyl source term available for long-range transport
}

\author{
X. Davoine ${ }^{1}$ and M. Bocquet ${ }^{1,2}$ \\ ${ }^{1}$ CEREA, Joint Laboratory École Nationale des Ponts et Chaussées/EDF R\&D, France \\ ${ }^{2}$ CLIME, Joint Project INRIA/École Nationale des Ponts et Chaussées, France
}

Received: 23 November 2006 - Published in Atmos. Chem. Phys. Discuss.: 2 January 2007

Revised: 14 March 2007 - Accepted: 14 March 2007 - Published: 21 March 2007

\begin{abstract}
The reconstruction of the Chernobyl accident source term has been previously carried out using core inventories, but also back and forth confrontations between model simulations and activity concentration or deposited activity measurements. The approach presented in this paper is based on inverse modelling techniques. It relies both on the activity concentration measurements and on the adjoint of a chemistry-transport model. The location of the release is assumed to be known, and one is looking for a source term available for long-range transport that depends both on time and altitude. The method relies on the maximum entropy on the mean principle and exploits source positivity. The inversion results are mainly sensitive to two tuning parameters, a mass scale and the scale of the prior errors in the inversion. To overcome this hardship, we resort to the statistical L-curve method to estimate balanced values for these two parameters. Once this is done, many of the retrieved features of the source are robust within a reasonable range of parameter values. Our results favour the acknowledged three-step scenario, with a strong initial release (26 to 27 April), followed by a weak emission period of four days (28 April-1 May) and again a release, longer but less intense than the initial one (2 May-6 May). The retrieved quantities of iodine-131, caesium-134 and caesium-137 that have been released are in good agreement with the latest reported estimations. Yet, a stronger apportionment of the total released activity is ascribed to the first period and less to the third one. Finer chronological details are obtained, such as a sequence of eruptive episodes in the first two days, likely related to the modulation of the boundary layer diurnal cycle. In addition, the first two-day release surges are found to have effectively reached an altitude up to the top of the domain $(5000 \mathrm{~m})$.
\end{abstract}

Correspondence to: $\mathrm{M}$. Bocquet

(bocquet@cerea.enpc.fr)

\section{Introduction}

On the 25 April 1986, 21:23 UTC, started in Chernobyl, Ukraine, the most devastating nuclear civil accident ever. One of the main challenges since the accident took place is to identify the source term.

Reconstructions of the accident source term have been previously carried out using core inventories, estimated by radionuclides kinetic models representing the core dynamics. Another source of information is the activity concentrations in air measured in Europe (but also in Asia and North America), and the deposited activity measurements, especially on Ukraine and Belarus. Using these data implies either using purely statistical approaches or back and forth confrontations between model simulations driven by meteorological data. These methods helped create a consensus on the source term, ventilated into many radionuclides. It is believed the error attached to this consensus might be as large as 50\%. This paper tackles a complementary approach: the reconstruction of the source term using inverse modelling techniques through a numerical transport model (or rather its adjoint in the present case).

\subsection{The Chernobyl source term}

In this study we are mainly interested in the radionuclides available for long-range transport, with a significant sanitary impact, that is: mainly iodine-131, but also caesium-134 and caesium-137. The source term of the fraction of these radionuclides which has been released into the atmosphere will be investigated here. Obviously this was very early a matter of concern for the community, because of the health impact of these radionuclides. First studies (the 1986 USSR report to IAEA and many other contributors mentioned later) gave good estimations of the total emitted activity and gave first assessments on the temporal profiles although not all of them were agreeing. However it was clearly emphasised that the

Published by Copernicus GmbH on behalf of the European Geosciences Union. 
release could have reached levels of the free troposphere, rising above $2000 \mathrm{~m}$ and possibly up to $4500 \mathrm{~m}$ in the initial explosion.

\subsubsection{Activity released in the atmosphere}

The main results on the source term obtained up to ten years after the accident were summarised in the 1995 OECD report (Waight et al., 1995) and references therein, and its 2002 update. This source term has been reprised many times since then (Brandt, 2002). In particular, it was estimated that $1.76 \times 10^{18} \mathrm{~Bq}$ of iodine-131, $8.5 \times 10^{16} \mathrm{~Bq}$ of caesium-137, and $5.4 \times 10^{16} \mathrm{~Bq}$ of caesium-134 were released.

\subsubsection{Temporal profile}

The temporal profile of emissions is necessarily a matter of greater uncertainty. The first estimation came from the initial USSR report and Persson et al. (1987); Albergel et al. (1988). Devell (1995) and later De Cort (1998) published finer estimations of the release history over the main ten-day release (later releases were of a much lower magnitude). The ten days period is segmented into three stages. The first one (first three days) corresponds to the initial explosion, with a fragmentation and release of a fraction of the fuel. The third one (last three days) corresponds to the emission due to the fuel fire, and the core melt-down. The second one (four days in between), weaker, makes the transition between the two main releases. An up-to-date estimation of the day-to-day release of iodine-131 is given in the 2000 UNSCEAR report (UNSCEAR, 2000). In that report, the third stage is not as intense as in the Waight et al. (1995) report, but the total amount is unchanged.

\subsubsection{Vertical profile}

The vertical profile is even more difficult to estimate. Many factors are to be taken into account: mechanical factors (explosion), heat, local meteorological factors (updraft by nearby front), local wet scavenging conditions, and obviously boundary layer cycles. In earlier papers (Gudiksen et al., 1989; Albergel et al., 1988), it was stressed that the first release must have reached $2000 \mathrm{~m}$ and more. A profile was proposed by Lange et al. (1988) and by Hass et al. (1990).

\subsection{The observations}

The observations used in this study are activity concentration measurements. As stressed earlier by Gudiksen et al. (1989), using deposited activity measurements for large scale inverse modelling purposes would be unwise. Indeed the uncertainty chain comprising precipitation estimation is longer and much less reliable than concentration measurements. Moreover, caesium-137 deposited by Chernobyl is a small fraction of the past nuclear tests fallout which makes the information difficult to extract. Data are provided by the REM-database at the Environment Institute (Joint Research Centre, Ispra, Italy). These are average values with very variable integration times, ranging from ten minutes to several hours, with a median of $11 \mathrm{~h}$. After a mild and unbiased filtration, 1274 measurements are used for iodine-131, 943 for caesium-134 and 1210 for caesium-137.

\subsection{Objective and outline}

The objective of this paper is not to determine the point of emission coordinates. This subject has been investigated on a different real case: ETEX (Bocquet, 2007). Therefore, Chernobyl location is supposed to be known a priori. Instead of the spatial coordinates, we are looking for the effective altitude and temporal release profile. Hence, one is looking for a two-dimensional field: $\sigma(z, t)$. The objective of this paper is to reconstruct the source term $\sigma(z, t)$ representing species available for long range transport with sizeable health impacts: mainly iodine-131, caesium-134 and caesium-137.

In Sect. 2, we briefly present the method and the tools it relies on: a numerical transport model, the adjoint of this model, and advanced inverse modelling techniques. In Sect. 3, we present the main results: a typical inversion outcome for iodine-131. The influence of the inversion parameters is discussed in details. Two such parameter values are determined using the L-curve method. Sensitivity studies to ascertain the typical result are described. Inversions on caesium-134 and caesium-137 are performed and the results are discussed. The conclusions are given in Sect. 4.

\section{The method}

\subsection{Numerical transport model}

The chemistry-transport model [CTM] POLAIR3D, which is employed for this study is a Eulerian numerical solver of the reactive dispersion equation for the concentration of the trace species $c$ (several species may be considered):

$\frac{\partial c}{\partial t}+\operatorname{div}(\boldsymbol{u} \nabla c)=\operatorname{div}\left(\rho \boldsymbol{K} \nabla\left(\frac{c}{\rho}\right)\right)-\Lambda^{\mathrm{s}} c-\Lambda^{\mathrm{d}} c+\sigma$

where $\boldsymbol{u}$ is the wind field, $\rho$ the air density, and $\sigma$ the source for this species. $\Lambda^{\mathrm{s}}$ is the wet scavenging coefficient and $\Lambda^{\mathrm{d}}$ represents the radioactive decay. $\boldsymbol{K}$ is the eddy diffusivity matrix, supposed to be diagonal. The vertical component is given by $K_{z}$ (the default parametrisation is the Louis parametrisation (Louis, 1998)). The horizontal component has a constant value $K_{H}$ (in this study, $K_{H}=0$ ). The boundary conditions have to be specified, in particular at ground:

$K_{z} \nabla c \cdot \boldsymbol{n}=v^{\mathrm{dep}} c$

where $\boldsymbol{n}$ is the upward oriented unitary normal vector. All other boundary conditions are taken null. 
A validation of this model on the specific case of passive tracers and radionuclides at regional scale has been performed and is described in (Quélo et al., 2007; Krysta, 2006). For a more detailed description of POLAIR3D, we refer to Boutahar et al. (2004). A general overview of the algorithms for the advection, diffusion, chemistry and splitting strategies used in the model is given by Verwer et al. (1998).

The space-time domain $\Omega$ chosen to simulate the Chernobyl accident and to compute the adjoint solutions needed later on, is $10.6^{\circ} \mathrm{W}-63.7^{\circ} \mathrm{E}$ and $34.4^{\circ}-69.3^{\circ} \mathrm{N}$ in space and from 25 April 1986 00:00 UTC to 9 May 1986 20:45 UTC (15 days) in time. A spatial resolution has been chosen: $1.125^{\circ} \times 1.125^{\circ}$ consistent with the ERA-40 ECMWF wind fields used in the computations. A finer discretisation has also been tested but will not be our main concern. There are 12 vertical layers which are separated by the following levels (relative altitude in metres): 0, 64, 236, 484, 796, 1184, 1616, 1984, 2616, 3184, 3616, 4384, and 5016.

\subsubsection{Some specific parametrisations}

\subsubsection{Radionuclides}

For the Chernobyl application, the only radionuclides taken into account are caesium and iodine. Two isotopes of caesium are modelled: ${ }^{134} \mathrm{Cs}$ and ${ }^{137} \mathrm{Cs}$. Iodine is known to be in many possible forms (elemental gas-phase iodine, organic methyliodine or particle-bound iodine). We only consider one lumped form for ${ }^{131} \mathrm{I}$, so that Eq. (1) is necessarily an approximation of the real micro-physics involved.

\subsubsection{Scavenging parametrisations}

Resistance models for dry deposition and microphysical parametrisations for wet scavenging are available in the model. Since the model is intended to inverse modelling application, we have chosen a very simple approach:

- a constant dry deposition velocity $v^{\mathrm{dep}}=0.2 \mathrm{~cm} \mathrm{~s}^{-1}$ for caesium, $v^{\mathrm{dep}}=0.5 \mathrm{~cm} \mathrm{~s}^{-1}$ for iodine, in the range of what is usually advised;

- two wet scavenging parametrisations are used. For the Belot scavenging type, the scavenging coefficient is parametrised as $\Lambda^{\mathrm{s}}=A p_{0}^{B}$ with $A=8.10^{-5}, B=0.8$ and $p_{0}$ the rain intensity (in $\mathrm{mm} / \mathrm{h}$ ). This represents a below-cloud scavenging. Should the particles radius be of the order of the micron and below, the application of this parametrisation would be questionable. This is certainly the case for part of the caesium particulate distribution. The second approach is based on a relative humidity threshold, beyond which the atmosphere is scavenged. This threshold is taken to be $80 \%$, beyond which $\Lambda^{\mathrm{s}}=3.5 \times 10^{-5}\left(\mathrm{RH}-\mathrm{RH}_{t}\right) /\left(\mathrm{RH}_{s}-\mathrm{RH}_{t}\right)$, where $\mathrm{RH}_{t}$ is the threshold and $\mathrm{RH}_{s}$ is the saturation value $(100 \%)$. It is a subgrid parametrisation of the in cloud scavenging (nucleation scavenging) and has been proposed first by Pudykiewicz (1989).

We refer to Pudykiewicz (1989); Brandt (1998); Baklanov (1999); Baklanov and Sørensen (2001) for comprehensive studies of these parametrisations. Wet scavenging is expected to dominate dry deposition, at least for long-range transport scale (Brandt, 1998).

\subsubsection{Radioactive decay}

The radioactive decay chain is taken into account for the three isotopes. The following values (in $s^{-1}$ ) have been used: $\Lambda_{131 \mathrm{I}}^{\mathrm{d}}=9.9710^{-7}, \Lambda_{134}^{\mathrm{d}} \mathrm{Cs}=1.0810^{-8}$, and $\Lambda_{137 \mathrm{Cs}}^{\mathrm{d}}=7.3210^{-10}$, which correspond to lifetimes of 8 days, 2 years and 30 years for ${ }^{131} \mathrm{I},{ }^{134} \mathrm{Cs}$ and ${ }^{137} \mathrm{Cs}$, respectively. So that at the time-scale of the Chernobyl plume dispersion, only iodine-131 decay is relevant.

\subsection{Generating the adjoint solutions}

The direct dispersion problem relates the solution of Eq. (1) to the measurements, represented as a $d$-vector $\mu$. An activity concentration measurement (in $\mathrm{Bq} \mathrm{m}^{-3}$ ) may be formalised by an integral over the space-time domain $\Omega$ :

$\mu_{i}=\int_{\Omega} \mathrm{d} t \mathrm{~d} \boldsymbol{x} \rho(\boldsymbol{x}, t) \pi_{i}(\boldsymbol{x}, t) c(\boldsymbol{x}, t)+\varepsilon_{i}$,

where $\varepsilon_{i}$ is the error related to measurement $\mu_{i} . \pi_{i}(\boldsymbol{x}, t)$ is a sampling function which specifies how the activity is measured. To any $\mu_{i}, i=1, \cdots, d$ one can associate a solution $c_{i}^{*}$ of the adjoint transport model (Uliasz, 1983; Pudykiewicz, 1998):

$$
-\frac{\partial c_{i}^{*}}{\partial t}-\operatorname{div}\left(\boldsymbol{u} \nabla c_{i}^{*}\right)=\operatorname{div}\left(\rho \boldsymbol{K} \nabla\left(\frac{c_{i}^{*}}{\rho}\right)\right)-\Lambda^{\mathrm{s}} c_{i}^{*}-\Lambda^{\mathrm{d}} c_{i}^{*} .
$$

In the context of Chernobyl, the boundary conditions for $c_{i}^{*}$ are taken to be null provided the domain $\Omega$ be large enough. At the ground, the following boundary condition is specified

$$
K_{z} \nabla c_{i}^{*} \cdot \boldsymbol{n}=v^{\mathrm{dep}} c_{i}^{*} .
$$

Two examples of adjoint solutions are given in Fig. 1. They are not the complete 4-D solutions but their 2-D traces at the Chernobyl site. One is related to an activity concentration measurement on ${ }^{137} \mathrm{Cs}$ in Attikis, Greece, on 4 May 08:00 UTC (24 h-long), the other to an activity concentration measurement on ${ }^{131} \mathrm{I}$ in Paris, France, on 4 May at 09:00 UTC (24 h-long). With this definition one can show (see for example Hourdin and Talagrand (2006)) that $\mu_{i}$ can be formally related to $\sigma(\boldsymbol{x}, t)$ by the dual relation:

$\mu_{i}=\int_{\Omega} \mathrm{d} t \mathrm{~d} \boldsymbol{x} \rho(\boldsymbol{x}, t) c_{i}^{*}(\boldsymbol{x}, t) \sigma(\boldsymbol{x}, t)+\varepsilon_{i}$. 

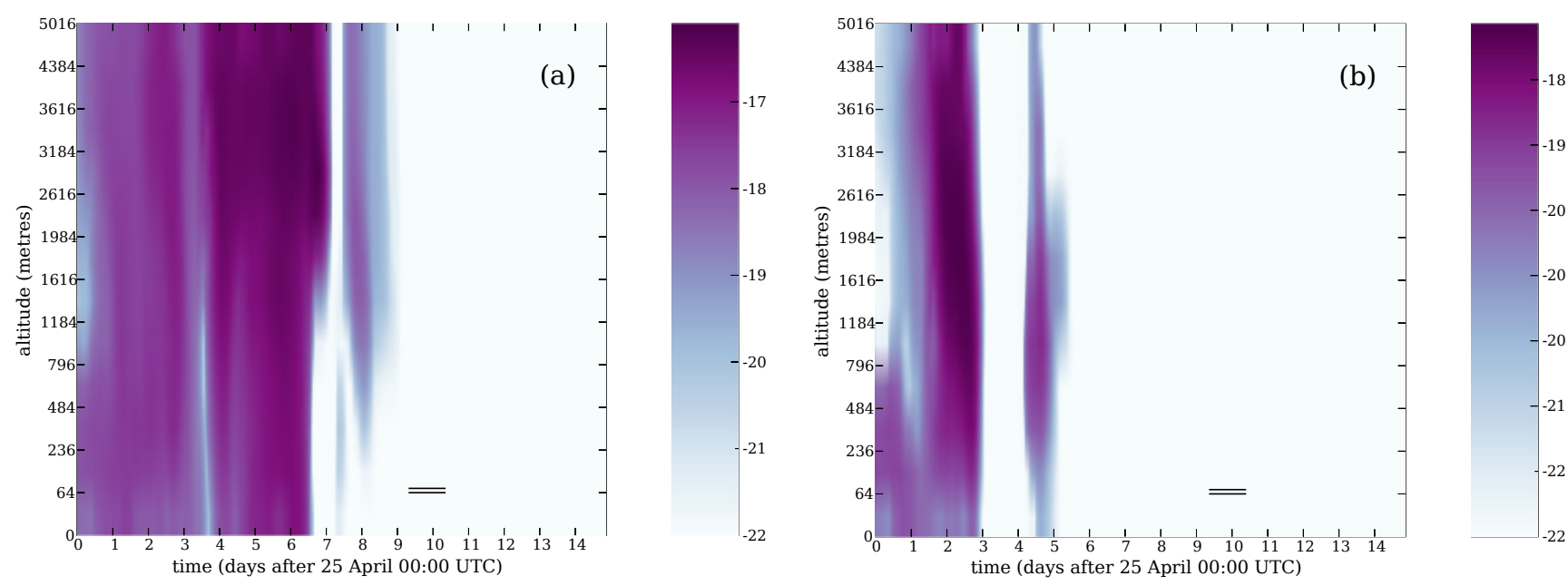

Fig. 1. Two examples of adjoint solutions traces at the Chernobyl power plant site. The observation period is indicated with a double line. Panel (a) is related to a measurement in Greece, panel (b) to a measurement in France.

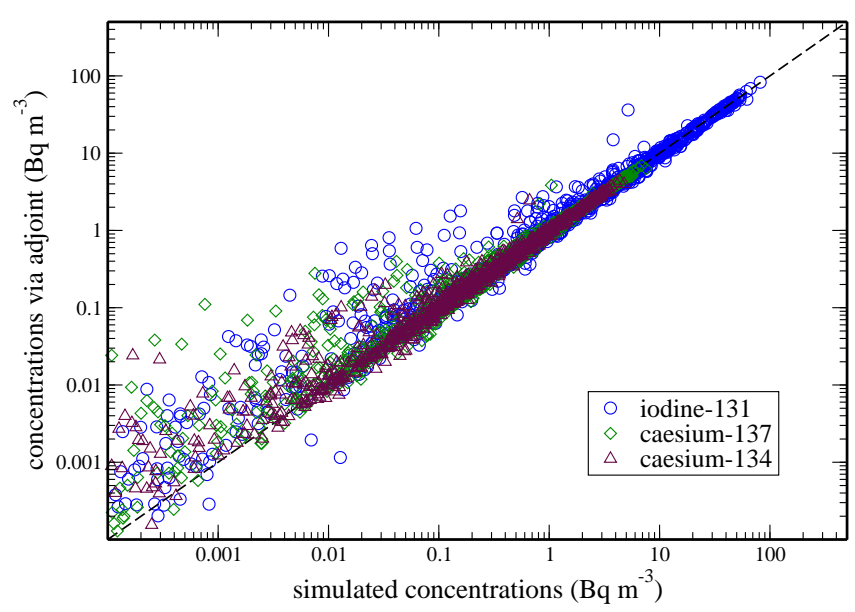

Fig. 2. This scatterplot compares simulated activity concentrations for all three species against activity concentrations obtained from the adjoint solutions. The Chernobyl source term used for this test is taken from Brandt (2002). The observations are those used in the inversions.

\subsection{The inverse problem}

\subsubsection{Posing the inverse problem}

From now on, we take the air density to be locally uniform, so that

$\operatorname{div}(\boldsymbol{u})=\mathbf{0}$.

In the case of the ETEX-I and Chernobyl simulations, we have checked that taking into account the stratification of air density has little influence on ground concentrations. So that $\operatorname{div}(\boldsymbol{u})=\mathbf{0}$ is a reasonable approximation for the dispersion tool at hand. Yet, this may have been wrong if a proper convection scheme had been implemented in the numerical model.

A discretisation of the continuous transport equation Eq. (1), leads to the numerical model. A similar discretisation of the continuous transport equation Eq. (4) leads to the numerical adjoint model. However, in general, this numerical adjoint model is not the adjoint of the numerical model, but rather an approximation. The discrepancy can be visualised on Fig. 2 which pictures a duality test. This test compares the measurement obtained from the direct approach Eq. (3) to the measurements obtained from the adjoint approach Eq. (6). We consider this approximation to be sufficient for our purpose. It has one major advantage: the computation of the adjoint solutions makes use only of the forward model, run backward, and with reverse wind-fields (Issartel and Baverel, 2003). As a statistical test (considering all measurements), the fractional bias between the concentration obtained via the adjoint or a direct simulation is greater than $50 \%$ for $12 \%$ of the observations, and greater than $20 \%$ for $27 \%$ of the observations. This was obtained for the Belot scavenging scheme. The result is the same for the relative humidity scheme.

In addition, for adjoint solutions and simulations computed on a finer grid $\left(0.5625^{\circ} \times 0.5625^{\circ}\right)$, the tests improve to a fractional bias greater than $50 \%$ for $7 \%$ of the observations, and greater than $20 \%$ for $19 \%$ of the observations. Yet, the coarse resolution adjoint solutions will be preferably used because they are consistent with the ERA-40 wind field resolution, and a proper averaging of the local scavenging schemes. 
The discretised version of the dual measurement equation is

$\mu_{i}=\sum_{k=1}^{N} c_{i, k}^{*} \sigma_{k}+\varepsilon_{i}$

where $k=1, \cdots, N$ runs on the grid-cell index. Volume elements of each grid-cell have been incorporated in $\sigma_{k}$, so that $\sigma_{k}$ represents the total activity (in $\mathrm{Bq}$ ) released in grid-cell $k$. If one defines the matrix $\boldsymbol{H}$ with entries $[\boldsymbol{H}]_{i, k}=c_{i, k}^{*}$, and $\varepsilon$ as the $d$-vector of errors, then the measurement equation reads:

$\mu=\boldsymbol{H} \sigma+\varepsilon$,

which embodies the inverse problem to be solved for.

\subsubsection{The inversion grid}

Because the accident location is known, the inversion grid is two-dimensional (time and altitude) as opposed to the fourdimensional grid used to compute the adjoint solutions. The finest inversion grid will incorporate the 12 layers of the original grid. The time and altitude part of both grid could in principle be different. The finest time resolution $\Delta_{t}$ will be $15 \mathrm{~min}$, though most of the inversion are performed at a resolution of $1 \mathrm{~h}$. For $\Delta_{t}=15 \mathrm{~min}$ and 12 layers, the total number of unknown variables is 13824 for one species and a 12-day period. This should be compared to about thousand measurements per species. It emphasises the ill-posed nature of the inverse problem at worse, or its ill-conditioned nature at best (for a larger $\Delta_{t}$ ). The time domain for the inversion starts at 25 April 21:00 UTC, a few minutes before the accident actually started, which will be denoted $t_{0}$ in the rest of the paper. It ends 12 days later.

\subsection{Dual variational approach}

\subsubsection{The method}

The most classical approach to solve Eq. (9), is to resort to a least-square criterion (possibly weighted by an observation error covariance matrix). It amounts to minimising the $1_{2}$ norm of the departure $\boldsymbol{\mu}-\boldsymbol{H} \boldsymbol{\sigma}$. However the number of variables could be far superior to the number of observations. Moreover, observation errors and model errors are reducing the information content of these observations. Therefore one needs a regularisation procedure, which picks up the most plausible potential source among those satisfying the observation constraints. To do so, it is usual to associate a regularising Tikhonov term to the error departure so that the complete cost function to minimise is

$\mathcal{L}(\boldsymbol{\sigma})=\frac{1}{2}(\boldsymbol{\mu}-\boldsymbol{H} \boldsymbol{\sigma})^{\dagger} \boldsymbol{R}^{-1}(\boldsymbol{\mu}-\boldsymbol{H} \boldsymbol{\sigma})+\frac{1}{2} \boldsymbol{\sigma}^{\dagger} \boldsymbol{B}^{-1} \boldsymbol{\sigma}$,

where $\boldsymbol{R}$ is the observation error covariance matrix, and $\boldsymbol{B}$ is the background error covariance matrix which contains the a priori known statistical information on the source. The $\dagger$ symbol denotes vector or matrix transposition. This cost function is equivalent to $4 \mathrm{D}$-Var (time and space components are all solved for altogether) albeit with a linear model of evolution. Such an optimisation problem can be equivalently solved in the physical space of measurements of lower dimensionality $d$. The dual cost function is

$$
\widehat{\mathcal{L}}(\boldsymbol{\beta})=\frac{1}{2} \boldsymbol{\beta}^{\dagger}\left(\boldsymbol{H} \boldsymbol{B} \boldsymbol{H}^{\dagger}+\boldsymbol{R}\right) \boldsymbol{\beta}-\boldsymbol{\beta}^{\dagger} \boldsymbol{\mu},
$$

and should be minimised on $\beta \in \mathbb{R}^{d}$. The optimal source and diagnosed errors are then given by

$\overline{\boldsymbol{\sigma}}=\boldsymbol{R} \boldsymbol{H}^{\dagger} \overline{\boldsymbol{\beta}}, \quad \overline{\boldsymbol{\varepsilon}}=\boldsymbol{\mu}-\boldsymbol{H} \overline{\boldsymbol{\sigma}}=\boldsymbol{R} \overline{\boldsymbol{\beta}}$,

where $\overline{\boldsymbol{\beta}}=\min _{\boldsymbol{\beta}} \widehat{\mathcal{L}}(\boldsymbol{\beta})$. This solution identifies with the minimum which could be obtained from criterion Eq. (10).

Yet, there are more efficient cost functions which could help solve Eq. (9). The maximum entropy on the mean formalism is a variant of the Bayes approach which allows to take into account prior information, such as the source positivity. The principle has been laid by Jaynes (1957a,b). It has been first used in geophysics by Rietsch (1977), considering a small number of constraints. It has been shown that such techniques are efficient in the case of an accidental event like Chernobyl (Bocquet, 2005b,c, 2007; Krysta and Bocquet, 2007), and they have been extended to the framework of geophysical data assimilation.

The implementation of the principle relies on the minimisation of the information content that separates the a posteriori probability density function of the source and the errors $p(\boldsymbol{\sigma}, \boldsymbol{\varepsilon})$ from the a priori distribution $v(\boldsymbol{\sigma}, \boldsymbol{\varepsilon})$, with the exception of the information provided by the observations. The discrepancy is measured by the relative entropy

$\mathcal{K}(p, v)=\sum_{\boldsymbol{\sigma}, \boldsymbol{\varepsilon}} p(\boldsymbol{\sigma}, \boldsymbol{\varepsilon}) \ln \left(\frac{p(\boldsymbol{\sigma}, \boldsymbol{\varepsilon})}{v(\boldsymbol{\sigma}, \boldsymbol{\varepsilon})}\right)$,

where the sum (possibly an integral) runs on all potential sources and errors. With the observations properly taken care of by Lagrange multipliers $\boldsymbol{\beta}$, one has to minimise on $p$ the functional

$\widetilde{\mathcal{L}}(p)=\mathcal{K}(p, v)+\boldsymbol{\beta}^{\dagger}\left\{\boldsymbol{\mu}-\sum_{\boldsymbol{\sigma}, \boldsymbol{\varepsilon}} p(\boldsymbol{\sigma}, \boldsymbol{\varepsilon})(\boldsymbol{H} \boldsymbol{\sigma}+\boldsymbol{\varepsilon})\right\}$,

which leads to the effective cost function in the dual space of the $\boldsymbol{\beta}$

$\widehat{\mathcal{L}}(\boldsymbol{\beta})=\ln \left\{\sum_{\boldsymbol{\sigma}, \boldsymbol{\varepsilon}} v(\boldsymbol{\sigma}, \boldsymbol{\varepsilon}) \exp \left(\boldsymbol{\beta}^{\dagger}(\boldsymbol{H} \boldsymbol{\sigma}+\boldsymbol{\varepsilon})\right)\right\}-\boldsymbol{\beta}^{\dagger} \boldsymbol{\mu}$.

The minimum of this effective cost function determine completely the optimal $p$. An estimator of the source and the errors can then be obtained from it. The most convenient estimator turns out to be the average. 
As is clear from Eq. (15), the actual form of the cost function that results from the technique depends on the a priori. When the source prior is Gaussian, and when the observation errors are also Gaussian, then the cost function is a leastsquare cost function of the form Eq. (11). The error prior statistics will be assumed Gaussian from now on.

Two other types of source priors are used in this study. They are both positive laws. At first, it is assumed a priori that a grid-cell $k$ emits a mass $m_{k}$ of activity, with a probability $\gamma_{k} \cdot 1-\gamma_{k}$ is the probability that it emits nothing. This is a Bernoulli-type law. It is a bounded law. The formalism then leads to the cost-function

$$
\begin{aligned}
\widehat{\mathcal{L}}(\boldsymbol{\beta})= & \sum_{k=1}^{N} \ln \left\{1-\gamma_{k}+\gamma_{k} \exp \left(m_{k}\left[\boldsymbol{\beta}^{\dagger} \boldsymbol{H}\right]_{k}\right)\right\} \\
& +\frac{1}{2} \boldsymbol{\beta}^{\dagger} \boldsymbol{R} \boldsymbol{\beta}-\boldsymbol{\beta}^{\dagger} \boldsymbol{\mu} .
\end{aligned}
$$

The optimal source is then given by

$\bar{\sigma}_{k}=m_{k} \frac{\gamma_{k} \exp \left(m_{k}\left[\overline{\boldsymbol{\beta}}^{\dagger} \boldsymbol{H}\right]_{k}\right)}{1-\gamma_{k}+\gamma_{k} \exp \left(m_{k}\left[\overline{\boldsymbol{\beta}}^{\dagger} \boldsymbol{H}\right]_{k}\right)}$,

and the analysed errors are still given by $\overline{\boldsymbol{\varepsilon}}=\boldsymbol{R} \overline{\boldsymbol{\beta}}$. The second type of law is the Poisson-like law. If $m_{k}$ is a reference activity value, grid-cell $k$ has a probability $e^{-\theta_{k}}\left(\theta_{k}\right)^{x_{k}} /\left(x_{k}\right.$ !) to emit $x_{k} m_{k}$, where $x_{k}$ is an integer. $\theta_{k}$ plays a similar role to $\gamma_{k}$. It is not a bounded law, so that peaks of release are allowed, though with a low probability. The formalism then leads to the cost-function

$$
\begin{aligned}
\widehat{\mathcal{L}}(\boldsymbol{\beta})= & \sum_{k=1}^{N} \theta_{k}\left\{\exp \left(m_{k}\left[\boldsymbol{\beta}^{\dagger} \boldsymbol{H}\right]_{k}\right)-1\right\} \\
& +\frac{1}{2} \boldsymbol{\beta}^{\dagger} \boldsymbol{R} \boldsymbol{\beta}-\boldsymbol{\beta}^{\dagger} \boldsymbol{\mu} .
\end{aligned}
$$

The optimal source is then given by

$\bar{\sigma}_{k}=m_{k} \theta_{k} \exp \left(m_{k}\left[\overline{\boldsymbol{\beta}}^{\dagger} \boldsymbol{H}\right]_{k}\right)$.

On can show that the Poisson prior is a limiting case of the Bernoulli prior when $\bar{\sigma}_{k} / m_{k}$ and $\gamma_{k}$ tend to zero. So that taking the Bernoulli mass scale $m$ to large values, while keeping $\gamma$ low enough ensures that the Bernoulli prior tends to a Poisson prior.

\subsubsection{The parameters controlling the inversions}

We would like to stress the meaning and importance of the parameters controlling the priors

- $m_{k}$ is an a priori (activity) scale for cell $k$. The set of $m_{k}$ does not determine the total reconstructed mass but the latter is necessarily sensitive to the $m_{k}$ if the information content of the measurements is insufficient. $A_{\mathrm{s}}$ is the total estimated activity released by the Chernobyl accident for species s, given by earlier reports. The values $A_{131}, A_{137} \mathrm{Cs}$, and $A_{134} \mathrm{Cs}$ are those given in (Waight et al., 1995). Then we shall note: $m_{\mathrm{s}}^{0}=A_{\mathrm{s}} / N$, that is $m_{\mathrm{s}}^{0}$ is the prior average released activity per grid-cell. The a priori parameter $m_{k}$ for species $\mathrm{s}$ will be defined in terms of $m_{\mathrm{s}}^{0}$. Obviously, $m_{k} / m_{\mathrm{s}}^{0}$ has to be much larger than 1 to avoid any unrealistic uniform solutions. We do not want to favour a priori any location in altitude or in time. Then it is natural to ascribe the same scale $m$ (in units of $m_{\mathrm{s}}^{0}$ ) to all grid-cells. However the vertical spacing is not uniform and the grid-cell volumes are unequal. As a consequence, one needs to correct the typical mass scale $m$ to $m_{k}=\frac{H_{k}}{H} N_{z} m$, where $H$ is the vertical extent of the domain, $H_{k}$ is the height of cell $k$, and $N_{z}$ is the number of vertical layers. Later on, the mass $m$ (in units of $m_{\mathrm{s}}^{0}$ ), will be sufficient to describe an inversion, since the $m_{k}$ derive from $m$. In the case where the source prior stems from a Bernoulli assumption, then $m_{k}$ corresponds to the maximum activity released in cell $k$.

- A very low $\gamma_{k}$, or $\theta_{k}$, is to be used in the context of an accidental source reconstruction, where absence of emission is the norm. They will be both taken uniform so that $\gamma_{k} \equiv \gamma$, and $\theta_{k} \equiv \theta$.

- Other crucial parameters are the error covariances that parametrise $\boldsymbol{R} . \boldsymbol{R}$ is taken to be diagonal with $[\boldsymbol{R}]_{i i}=\chi_{i}$. For a uniform treatment of the errors, $\chi_{i} \equiv \chi$. The uniform variance hypothesis stipulates that all measurements have the same prior error standard deviation. It is rather simple to implement, but it grants high credit to the greater measurements, and less credit to the lower (especially those lower than the standard deviation, which are effectively considered as noise). It can also be assumed that the errors could be relative to the measurement amplitude, so that $\chi_{i}$ is alternatively chosen a priori as a fraction of the measurement $\mu_{i}$, provided $\mu_{i}$ is above a threshold. $\chi$ and the $\chi_{i}$ are obviously crucial since they balance the relative weights of the background and observation terms.

- The retrievals also depend crucially on the inversion grid, since the latter determine the number of unknown variables. In Bocquet (2005a), it has been shown that a reconstruction may end up in a complete failure, that no regularisation would prevent, when the resolution is too fine. In the particular case where the location of the source has been determined before any reconstruction, it has been shown that such a breakdown should not occur. Consequently, in this context, a too high resolution reconstruction should at worst be useless and inefficient, but not detrimental to the retrieval. 


\section{Results}

In the following will be given our main results. There is no unique solution since the inversions depend on the priors on the source and the errors, and the quantitative parameters that describe them. Nonetheless, in order to constrain the solutions, a parameter selection technique known as the L-curve method will be used to assess the mass scale and variances which parametrise the priors. One will then be looking for robust features that appear recurrently in the reconstructions. We will firstly describe what could be considered as a typical result for iodine-131. The priors parameters will be assessed and several sensitivity tests on the inversion settings are then performed. From these tests will emerge several robust features. A few comments will then be made on the information content of the observations. Eventually, a few results on caesium will be examined.

\subsection{Typical inversion for iodine 131}

The setting for what emerged as a typical inversion dedicated to iodine-131 is: $m=14$ (in units of $m_{131}^{0}$ ), $\gamma=10^{-9}$, and a uniform prior error with standard deviation of $0.25 \mathrm{~Bq} \mathrm{~m}^{-3}$. The justification for these parameters values choice will be given below.

The time resolution is one hour and the vertical resolution is given by the 12 layers given above. The scavenging is based on the relative humidity scheme. A time-altitude density plot is given in Fig. 3a. The time axis starts on the 25 April 1986 00:00 UTC. The temporal profile is given by the total activity column for each one hour period. The corresponding graph is represented in Fig. $3 b$.

\subsubsection{Total released activity}

The total released activity obtained in this retrieval is $1.6210^{18} \mathrm{~Bq}$, close to the consensus estimation of $1.7610^{18} \mathrm{~Bq}$. Please note that we do not apply any decay correction to figures.

\subsubsection{Three-stage scenario}

The retrieval agrees well with the three-stage scenario. The first stage corresponds to a strong release in altitude, from the ground to the top of the domain $(5016 \mathrm{~m})$. The first surge, matching the initial explosion, is diagnosed to have been efficient as a source for long-range transport above $800 \mathrm{~m}$. The second stage corresponds to a a weaker release. The third stage is characterised by a set of secondary source spots from 3 to 5 May, which are distributed from the ground up to $3500 \mathrm{~m}$.

\subsubsection{Robust events}

There are significant features, which could be qualified as robust and which are worth mentioning.
- In the first stage, there are three strong release traces in altitude. The first one starts at $t_{0}$, lasts for $8 \mathrm{~h}$ and extends from 800 to the top of the domain. This certainly corresponds to the initial explosion and release. This can be ascribed to the inversion uncertainty but also to a transport delay to the upper layers. The second starts at $t_{0}+15 \mathrm{~h}$ and rises up from $1200 \mathrm{~m}$ to the top. It coincides with the ignition of the combustible gas from the core and the start of the fuel fire (see UNSCEAR, 2000). It is followed by a third strong release from the ground up the top, which ends at $t_{0}+50 \mathrm{~h}$. The last two events are not well chronologically separated so that they could be two aspects of the same event but at different altitude. The three episodes could be the modulation of the same event by the boundary layer cycle, which would allow an efficient release of radionuclides to the higher layers for a fraction of the day only. So that the effective source (that we may called "secondary") for long-range transport would appear segmented.

- The second stage displays a much lower intensity peak. It is mainly effective at the top of the domain $(5016 \mathrm{~m}$ high). However the total released activity at this stage is consistent with the standard scenario.

- The third stage release displays several secondary sources. They look faint but are very robust to many changes in the parameters of the inversion. It starts at $t_{0}+7$ days (the 2 May at 18:00 UTC) and lasts for three days. It is consistent with the spread of the corium in the sub-reactor region and the vaporisation of water contained in pools, releasing a large quantity of aerosols.

The temporal extension of these events should be interpreted as the time period when the phenomenon has significant influence. It is widened in the inversion by the fact that the Bernoulli prior is imposing an upper bound. Here it is $m=14$, which is attained in several of the grid-cells where these events are significant. There are other features that often appear but which are less robust.

An histogram of the typical day-to-day release profile obtained from inversions is given in Fig. 4, and compared to other profiles estimated earlier. It is clear that our inversion apportionment favours the initial release period and weakens the third period as compared to earlier estimations.

This inversion, which we believe is typical, will be referred to as the reference case.

\subsection{Assessing the prior mass scale and variances}

\subsubsection{Strong sensitivity of iodine-131 reconstruction to} prior mass scale and prior variances

$m$ has been discussed as one of the parameters controlling the inversion. Ideally one would like the inversions not to depend on this parameter, which must be given a priori. Such 

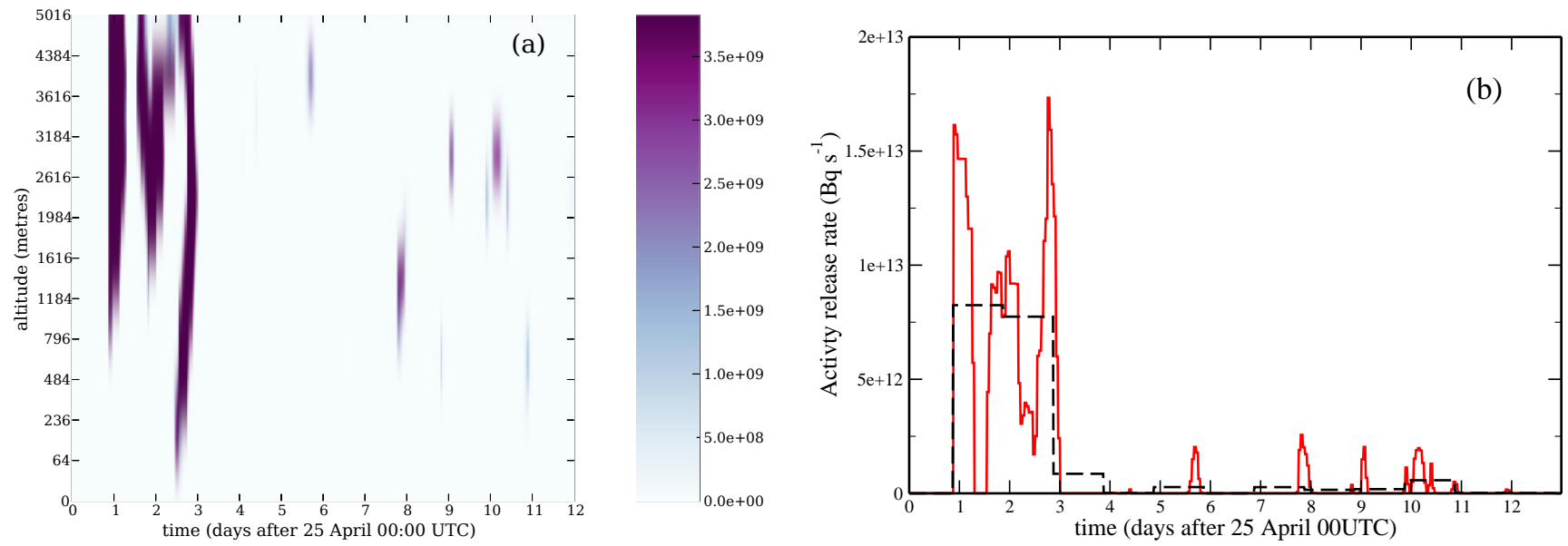

Fig. 3. A typical solution of the inversion problem based on a Bernoulli source prior, and a uniform Gaussian error prior. The left-hand-side panel (a) is a density graph representing the activity released per unit of time and unit of altitude (in $\mathrm{Bq} \mathrm{m}^{-1} \mathrm{~s}^{-1}$ ). The right-hand-side panel (b) are reconstructed hourly and day-to-day (dashed line) profiles of the iodine-131 release rate.

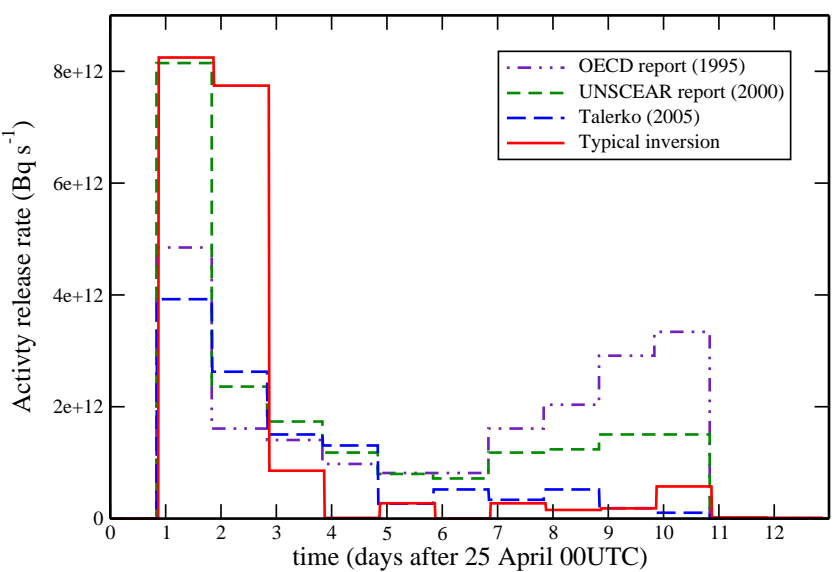

Fig. 4. Comparison of the day-to-day source term for iodine-131, in this study, in the 1995 OECD report, in the 2000 UNSCEAR report, and by Talerko (2005).

a scale is bound to appear in the settings since it gives away a breaking of symmetry due to the choice of a positive law. Hence this parameter is fundamental. In previous inversions performed on ETEX-I using such a law (Bocquet, 2007), the retrievals had a moderate dependence on $m$, and it did not appear as a real difficulty. In the case of Chernobyl, the total activity retrieved in the inversions is more sensitive to the scale. We believe this is due to the many scales present in the data (from a few $\mathrm{m} \mathrm{Bq}$ to hundreds of $\mathrm{Bq}$ ). To overcome this problem, one may resort to techniques devoted to (hyper-) parameter estimation: maximisation-expectation algorithm, unbiased predictive risk estimator, generalised cross validation, generalised maximum likelihood, etc. We have chosen the semi-empirical L-curve approach (Hansen, 1992), since it is (relatively) simple to implement, and is able to deal with a non-linear dependence on the parameter.

\subsubsection{L-curve approach}

The method compares the growth of the errors departure to the growth of the source departure (in a log-scale plot). The optimal parameter corresponds to the point of maximum curvature of the L-curve. Such a point is generally signalled by a corner or inflection in the log-scale plot of the departures. In this problem, the corners are not pronounced, but exist.

With $m$, the variances $\chi_{i}$ that determine the error priors are the parameters on which the retrievals depend the most. They tune the balance between the source and the errors prior, although in a less straightforward way than in 4D-Var. The two parameters are not independent, but they represent more than one degree of freedom. The cost functions have a non linear (or even quadratic) dependence on $m$. So that an estimation of $m$ is not a question that is widely discussed in applied statistics, whereas the dependence on $\chi$ is a more classical issue.

In Fig. 5a, is represented the L-curve on an inversion using $\gamma=10^{-9}$, with a fixed $\sqrt{\chi}=0.25 \mathrm{~Bq} \mathrm{~m}^{-3}$, and varying the mass scale $m$ from $m=5$ to $m=45$. The related scavenging scheme is the relative humidity. The maximum curvature (drawn in the inset figure) of the L-curve is attained at $m \simeq 14$.

As for $m$, parameter estimation can be invoked to estimate a balanced value for $\sqrt{\chi}$. Estimation techniques were primarily designed for this kind of parameter. We resort again to the L-curve approach. Assuming $m=14$, then the maximum curvature point is found at $\sqrt{\chi} \simeq 0.25 \mathrm{~Bq} \mathrm{~m}^{-3}$. The result is shown in Fig. 5b. 

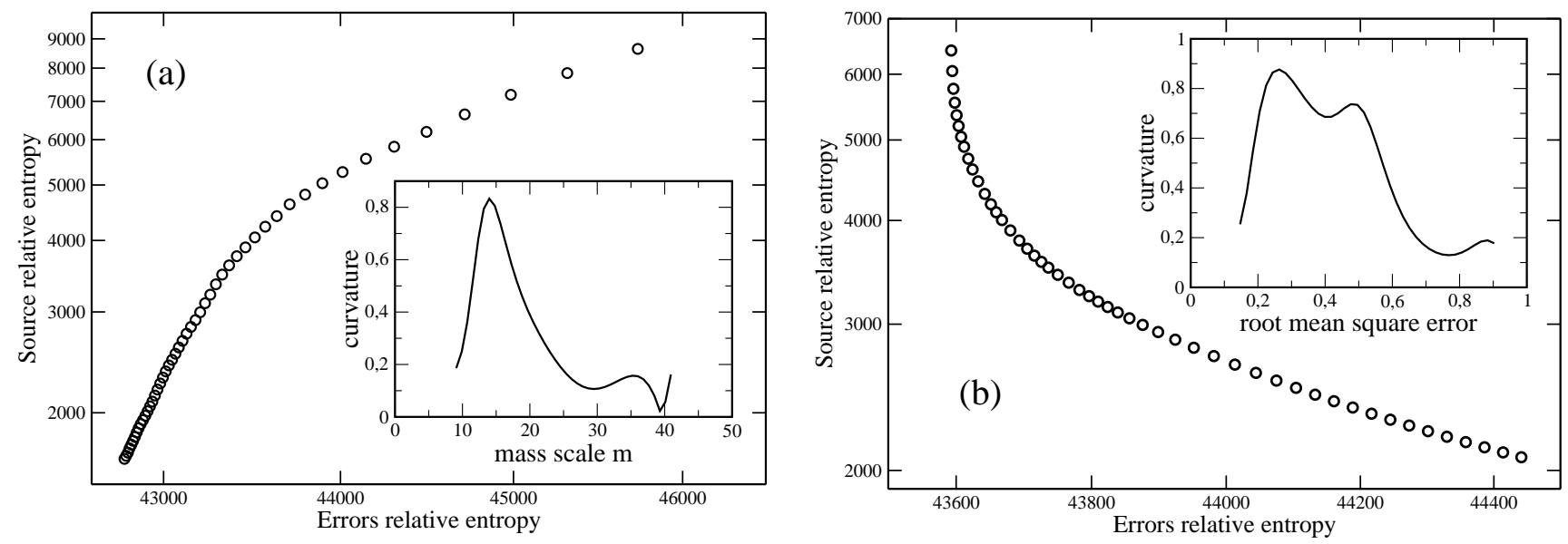

Fig. 5. On the left (a) is plotted the L-curve of ${ }^{131} \mathrm{I}$ inversion results when the mass scale is varied form $m=5$ to $m=45$, using $\sqrt{\chi}=0.25 \mathrm{~Bq} \mathrm{~m}^{-3}$. On the right $(\mathbf{b})$ is plotted the L-curve resulting from inversions when $\sqrt{\chi}$ is varied from $0.05 \mathrm{~Bq} \mathrm{~m}^{-3}$ to $1 \mathrm{Bqm}^{-3}$, using $m=14$.

Since the L-curve technique cannot easily be generalised to multidimensional analysis, the L-curve for $\chi$ assumes a prior value for $m$ and vice-versa. The solution couple $(m, \chi)$ is attained iteratively thanks to the existence of a fixed point. Taking this route, $\sqrt{\chi}=0.25 \mathrm{~Bq} \mathrm{~m}^{-3}$ and $m=14$ appears as a fixed point for iodine-131 inversions. This explains a posteriori why $\sqrt{\chi}=0.25 \mathrm{~Bq} \mathrm{~m}^{-3}$ and $m=14$ have been prescribed in Fig. 5.

The estimated $m$ will be shown to depend strongly on the type of inversion and species. Since $m$ is given in units of the previous estimation activity releases per grid-cell, it demonstrates that $m$ is not so much a scale for the total released activity, but also a measure of the fluctuations in the solution. The higher $m$, the stronger the peaks in the release as compared to the average release. This will be checked later on the caesium-134 and caesium-137 cases.

\subsection{Sensitivity of iodine-131 reconstruction to other inver- sion settings}

\subsubsection{Changing wet scavenging parametrisation}

Most of the parameters considered in this paper are related to the inversion setting. Varying physical parametrisations would also have a presumably strong influence on the retrievals. As advocated in (Brandt, 2002), one of the most influential physical process is the wet scavenging. That is why both Belot scavenging and a relative humidity scavenging were tested. The solution depicted in Fig. 6 is equivalent to the previous inversion for all parameters but the wet scavenging, which is Belot-type. A bigger total activity of $1.82 \times 10^{18} \mathrm{~Bq}$ is retrieved. As is clearly seen on the source profiles, there is little difference except for the two events on the 29 and 30 April which are diagnosed high in altitude in the Belot-based inversion. Since the relative humidity represents in-cloud scavenging as opposed to the Belot scheme (below-cloud scavenging), those two spots are likely to be scavenged areas with the relative humidity scheme. There are therefore less likely spots for a source for a model based on the relative humidity.

This analysis stresses the differences between two schemes. More importantly it emphasises the fact that not only does an inversion depend on the physical processes that affect a mass of radionuclides on its way from the source to the receptor, but it also strongly depends on the physical events that affect pollutants locally at the source site, and presumably at the receptor site too.

\subsubsection{Influence of uniform and relative errors prior}

As a hyper-parameter of the inverse problem, the standard deviation has been set to $0.25 \mathrm{~Bq} \mathrm{~m}^{-3}$. As a comparison, the measurements span from $10^{-4} \mathrm{~Bq} \mathrm{~m}^{-3}$ to $100 \mathrm{~Bq} \mathrm{~m}^{-3}$, with a median of $1.5 \mathrm{~Bq} \mathrm{~m}^{-3}$. The standard deviation was assumed uniform, which is certainly questionable.

A relative error prior has also been tested. It may sound as an appropriate prior for such an observable since an activity concentration may scale over several order of magnitudes. Yet, by construction, such a prior gives less credit to greater measurements which have potentially a strong information content. We observe that the robust spots that have been mentioned earlier are still present, even though their magnitude is different. These inversions seem nevertheless less reliable than with a uniform prior. As an example, $m$ was kept equal to 14 , then the errors were specified by a fraction $f$ of the measurements. A threshold $\tau$ was also assumed so as not to give too much credit to very low measurements. Three hyper-parameters were too many, and we 

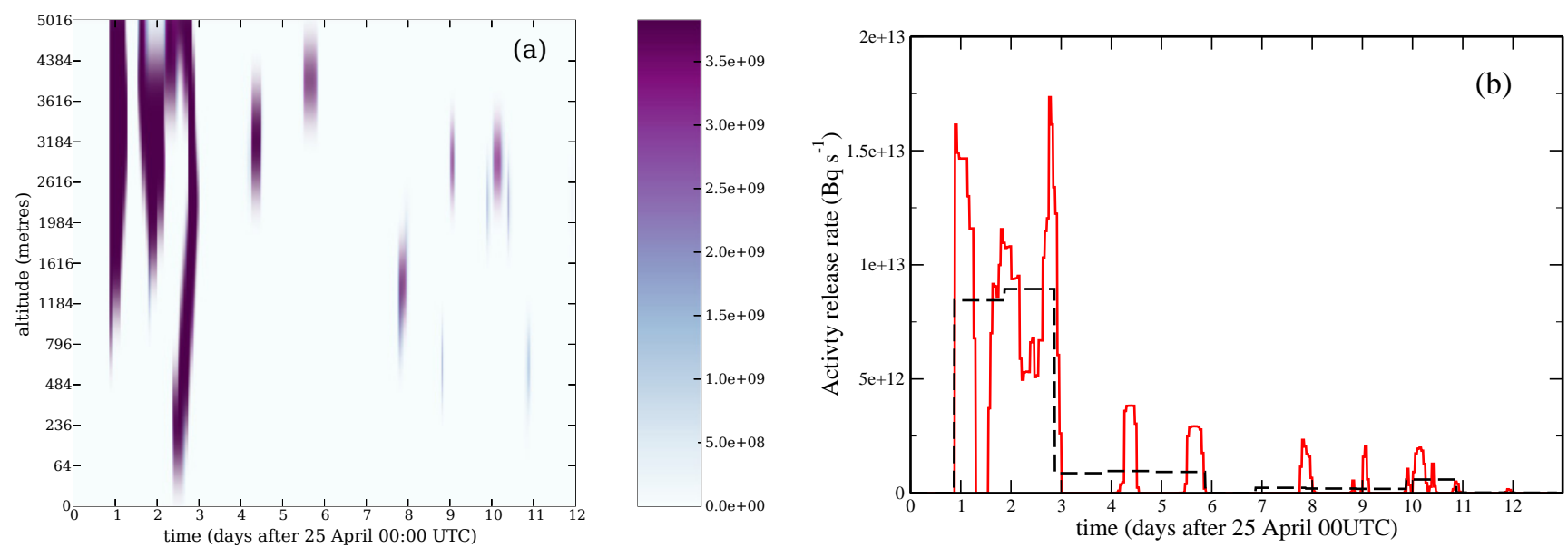

Fig. 6. The same as in Fig. 3, but with a model using a Belot scheme for the scavenging.

chose arbitrarily $\tau=0.01 \mathrm{~Bq} \mathrm{~m}^{-3}$. Then an L-curve estimate gives $f \simeq 1 \%$. The total retrieved activity is $1.37 \times 10^{18} \mathrm{~Bq}$ (Belot) and $1.48 \times 10^{18} \mathrm{~Bq}$ (relative humidity). The Belot result is represented in Fig. 7. It is observed that the third stage displays a stronger release than with the uniform error prior inversion.

\subsubsection{Influence of the time resolution}

The time resolution is then varied. It is appropriate to determine how robust the features put forward so far are with respect to time. In Fig. 8, two density plots are given for the same inversion specifications as for the reference case but with the time resolution of $15 \mathrm{~min}$ and $4 \mathrm{~h}$. The total released activity in the high resolution case is $1.3010^{18} \mathrm{~Bq}$, whereas it is $1.90 \times 10^{18} \mathrm{~Bq}$ in the coarser case (relative humidity scheme for both resolutions). It is remarkable how robust most of the spots are. The only significant difference is the vertical extension of the release event on 3 May.

\subsubsection{Influence of the source prior}

\section{Poisson}

The boundedness of the Bernoulli prior is its strength and its weakness as it does not allow for release peaks greater than $m_{k}$ in cell $k$. Such a problem could in principle be avoided by the Poisson prior which allows for such peaks, albeit with a much lower a priori probability. Unfortunately, it comes with numerical instability problems, since the cost function Eq. (18) involves an exponential. These numerical difficulties force to choose a low scale $m$, so that the retrieved solution is more contrived and not reliable. Although the main features are still present, they are distorted.

\section{Gaussian}

A fully Gaussian approach (least square or 4D-Var) has the flaw that it does not rely on the positivity of the source. We have nevertheless performed these inversions. The results are unsatisfying. Only the first stage events are recognised. Besides the solution is oscillating and has negative values. This can be seen in Fig. 9. The covariance matrices are parametrised with $\boldsymbol{B}=m^{2} \boldsymbol{I}_{N}$ and $\boldsymbol{R}=\chi \boldsymbol{I}_{d}$. Note that, contrary to the previous cases, only one parameter matters, since parameters $m$ and $\sqrt{\chi}$ represent the same degree of freedom of the inverse modelling system. Besides, the mass scale $m$ represents the scale of fluctuations (second order moments) of the source, whereas in inversions based on a non-Gaussian source prior, it was representing the first moment (in addition to parametrising higher moments). An L-curve analysis has been performed to determine the optimal $m$, though other methods are available for the related quadratic cost function. We found that $m / \sqrt{\chi}=4.5$ is optimal. The total retrieved activity is $1.09 \times 10^{18} \mathrm{~Bq}$, but it is a combination of positive and negative contributions. By lowering $m$, which would then be non-optimal, one can diminish the areas of negative values. However, this will shrink the solution and the total retrieved activity would be significantly lower than the previously estimated activity.

\subsection{Information content of the observations}

\subsubsection{Uncertainty, vertical and temporal details of the source}

All observation sites are far from Chernobyl: they are located in Scandinavia, Greece, central and western European countries. Activity concentrations over Ukraine, Belarus and Poland, are not available in the REM database. This is a warning not to draw definitive conclusions from the results 

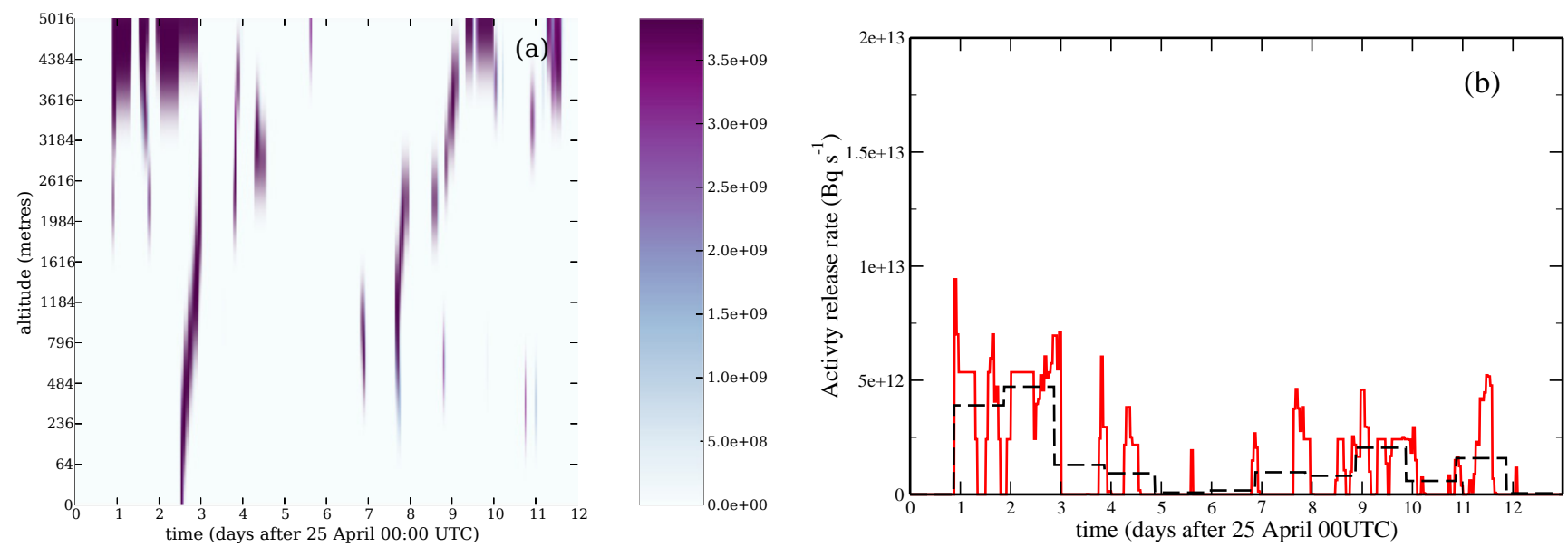

Fig. 7. The same as in Fig. 6, but with a relative error prior.
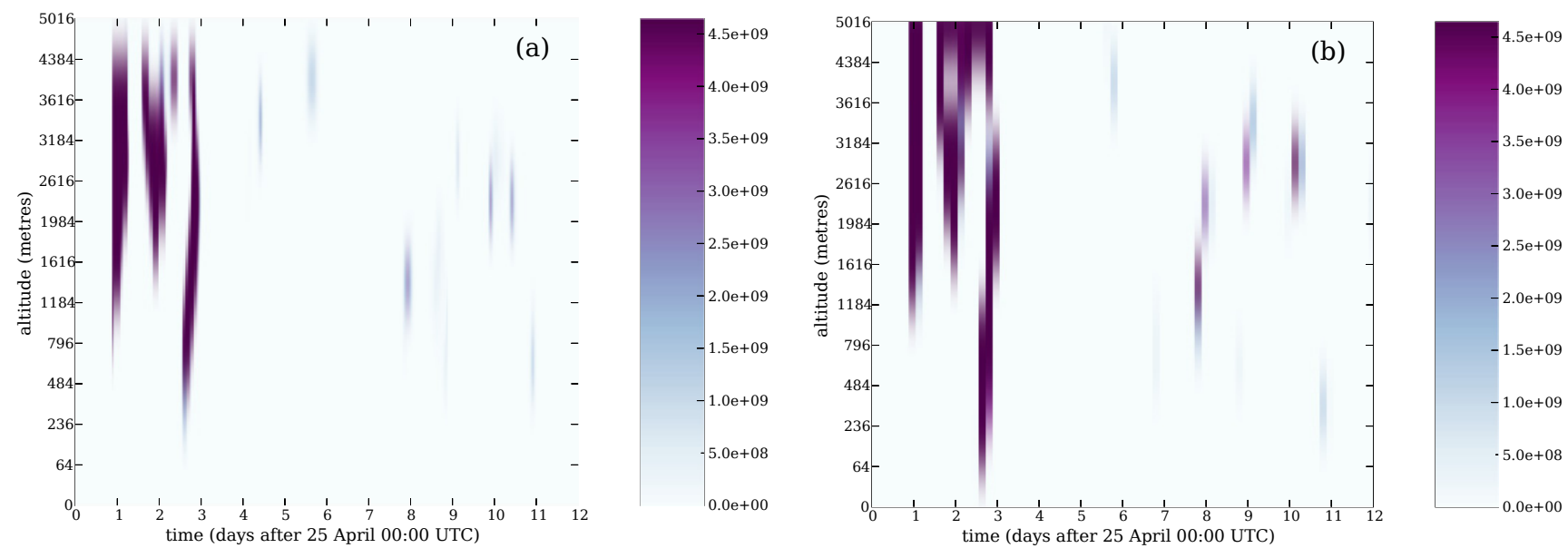

Fig. 8. Two examples of inversion with a higher time resolution ( $15 \mathrm{~min}$ in panel a) and a coarser time resolution ( $4 \mathrm{~h}$ in panel b).

obtained in the inversions. They cannot be too sensitive to fine details of the source. Indeed, one can assume qualitatively that on large space and time scale, the dispersion relation of transport equation is of the form $\omega \sqrt{-1}+\boldsymbol{k}^{\alpha}$ in Fourier space ( $\boldsymbol{k}$ is the wavevector), where $\alpha$ describes the nature of the effective diffusion ( $\alpha$ would be 2 for a Fickian diffusion). Therefore, $k_{z}^{-\alpha_{z}}$ is a cutoff for the measurement sensitivity to vertical details of the source, whereas their sensitivity to temporal details is attenuated by $\omega^{-1}$. When estimating surface concentrations from a column point-wise source, $\alpha_{z}$ is greater than 2 (mixing by wind fields acts effectively as superdiffusion). As a consequence, the reconstruction of the vertical details of the source is much more challenging than of the temporal ones. This is exemplified in Fig. 1 where the adjoint solutions exhibit draperies. All vertical reconstructions should therefore be interpreted with caution. Besides, the vertical retrieval is (as should be) strongly influenced by the numerical model representation of vertical diffusion (and therefore boundary layer dynamics). Even though vertical details should be handled cautiously, it does not invalidate the inversion: a purely temporal inversion can indeed be inferred by integrating out columns of ground and upper secondary sources.

\subsubsection{Observations informing part of the source domain}

Parameter $\gamma$ of the Bernoulli prior sets the probability of an emission in a given cell. For an accidental release inversion, or more generally when the mean of the emission is not known a priori, it is less committing to take it null. Taking $\gamma$ as low as $10^{-9}$ meets this purpose. For $\gamma \ll N^{-1}$, the inversions weakly depend on its actual value. Therefore, this parameter is of minor importance. It can serve however other purposes. 

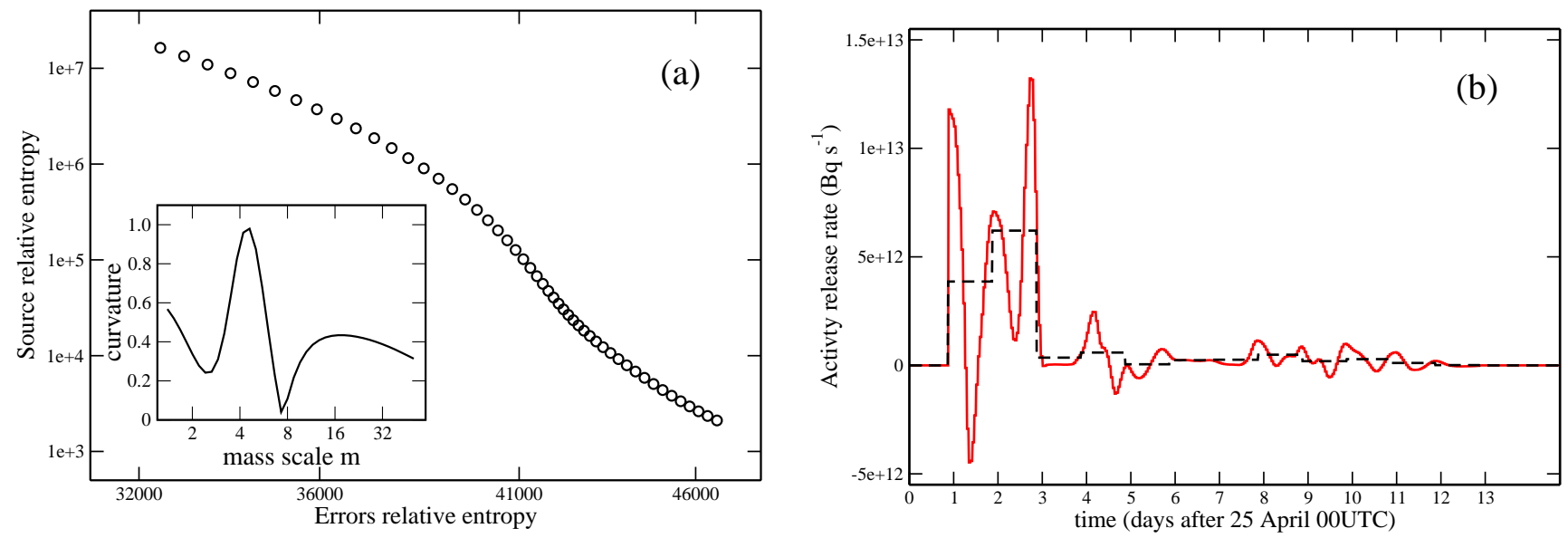

Fig. 9. On panel (b) is represented the temporal profile resulting from an iodine-131 inversion using Gaussian priors for both the errors and the source. The mass scale $m$ is estimated by the L-curve method: $m \simeq 4.5$ on panel (a). The L-curve obtained here has a shape which resembles more an L-curve based on the variation of $\sqrt{\chi}$, than an L-curve based on the variation of the mass scale $m$ of non-quadratic priors. This is explained by $\sqrt{\chi} \sim m^{-1}$.

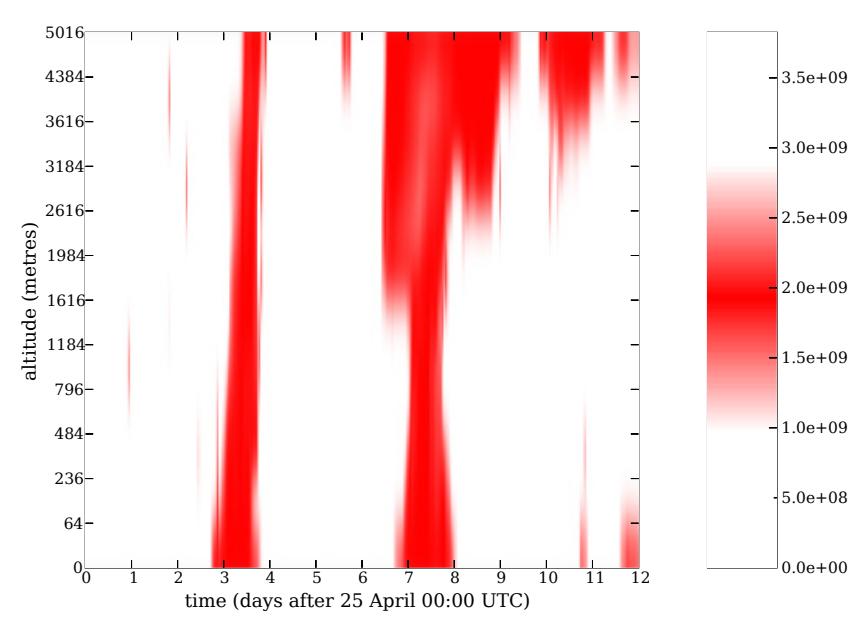

Fig. 10. An inversion on the reference case but with $\gamma=1 / 2$. The colour map is chosen so as to stress the mid-range source values close to $\gamma m$.

Generally speaking, the information content of an observation is not spread out uniformly on the inversion grid by the inversion procedure. The response of a measurement $\mu_{i}$ to a fluctuation of the source at position $z$ and time $t$, is given by the adjoint solution related to $\mu_{i}$

$\frac{\delta \mu_{i}}{\delta \sigma(z, t)}=c_{i}^{*}(z, t)$.

As can be seen with the adjoint solutions pictured in Fig. 1, a measurement will only give away significant information on a fraction of the domain.
Hence, the full set of observations used for an inversion is expected to shed light irregularly on the domain. There may be areas where the data actually tell little. In these areas, the inversion algorithm will yield the prior mean: $\gamma m_{k}$, that is to say a very low value. This can be exploited: set $\gamma=1 / 2$ instead of $\gamma=10^{-9}$, then these areas will be distinguished from areas that are truly predicted to be negligible from the observations. In turn, it is not possible to distinguish these areas from areas when the solution is predicted to be $m_{k} / 2$ from the observations. But the latter is less likely to occur in an accidental release context.

This idea is illustrated in Fig. 10 on the reference case but with $\gamma=1 / 2$. This has to be compared with Fig. 3. The areas where the data do not bring information are coloured. The third-stage surge is coated by an area of ignorance. A significant information blackout occurs on 2 May. There is also an information black-out on 28 April. As a practical conclusion, the retrievals on 28 April and on 2 May might underestimate the true release.

The REM data set used in the inversions presented in this paper is non-uniformly sensitive to the inversion grid-cells. Especially, only the measurements performed in Greece (14 measurements) are significantly sensitive to the part of the inversion grid supporting the third stage. This is illustrated in Fig. 11. Figure 11a displays the result of an inversion using only the observations from France (148 measurements). The area which is unaffected by these data and governed by the source prior appears in colour. Clearly, only the first five days are sensitive to this set. This set is not telling anything on the third stage. We have checked that the data from Greece are the principal set doing so. This is illustrated in Fig. 11b. Therefore, the estimation in the third stage from 

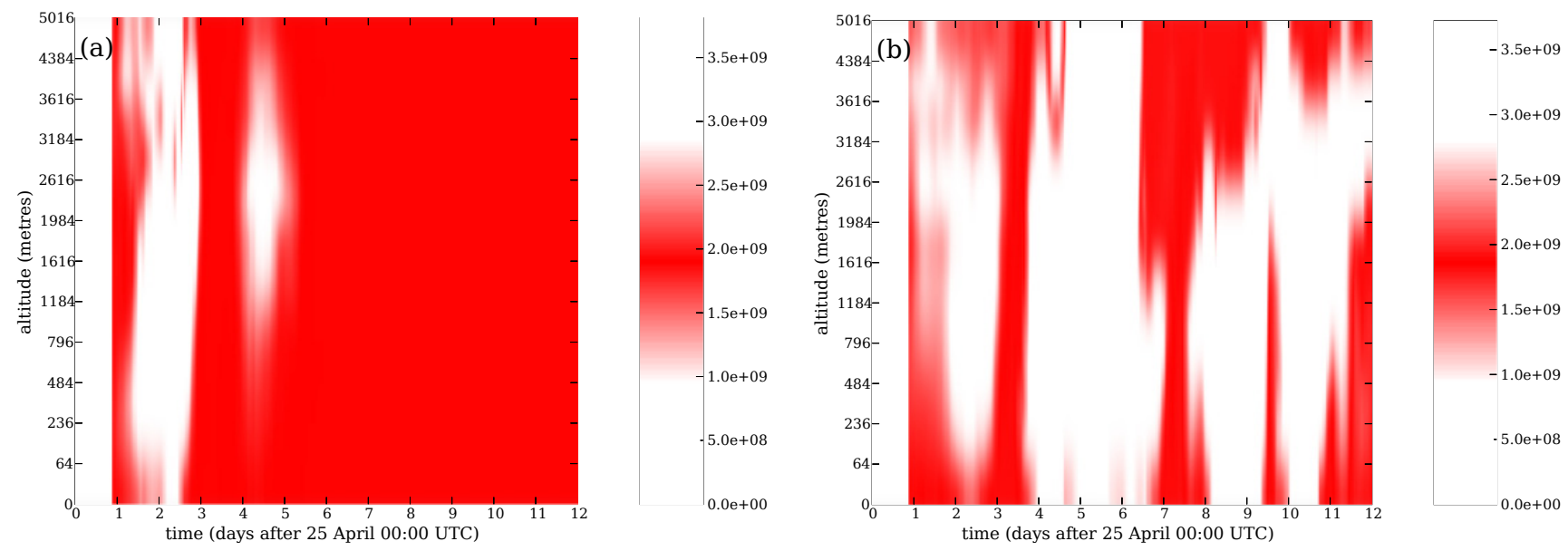

Fig. 11. The left hand side (a) is a reconstruction made out of the observations performed in France. The right hand side (b) is a reconstruction made out of the observations performed in Greece. The colour bar is purposely chosen as in Fig. 10.

inverse modelling relies on very few data, and should be taken with caution.

\subsection{Typical inversion for caesium-134 and caesium-137}

The same approach which was applied to iodine-131, can be applied to caesium-134 and caesium-137. The first step is to estimate the parameters: prior mass scale and prior error standard deviation. Their determination is more intricate than for iodine-131. The selection is operated by successive L-curves plotting on $m$ then on $\chi$, iteratively. For caesium-137, $m$ is estimated to lie in the range 45-75 (in units of $m_{137 \mathrm{Cs}}^{0}$ ), and $\sqrt{\chi}=0.10 \mathrm{~Bq} \mathrm{~m}{ }^{-3}$. The reconstruction is quite insensitive to a variation of $m$ within this range. So $m=60$ was chosen. The quite low value of the prior variance amounts to trusting the measurements and/or the model in the caesium-137 case more than in the iodine- 131 and caesium134 cases. The total released activity is $13.6 \times 10^{16} \mathrm{~Bq}$, to be compared to an earlier estimation of $8.5 \times 10^{16} \mathrm{~Bq}$. The relative mass scale $m$, compared to the iodine-131 case, indicates that the solution is more concentrated on higher peaks. This is illustrated in Fig. 12b, where the relative humidity scheme has been used.

For caesium-134, $m$ is estimated to 200 (in units of $m_{134 \mathrm{Cs}}^{0}$, and a relatively high $\sqrt{\chi}=0.40 \mathrm{~Bq} \mathrm{~m}^{-3}$. The total released activity is $3.5 \times 10^{16} \mathrm{~Bq}$, to be compared to an earlier estimation of $5.4 \times 10^{16} \mathrm{~Bq}$. The solution is clearly dominated by sharp peaks. It illustrates again that the parameter $m$ cannot only be interpreted as an indication of the total released activity but it also tells how sharp the solution is. For instance, if one trades the relative humidity scheme for the Belot scheme, then $m$ is estimated to be 130 and $\sqrt{\chi}=0.35 \mathrm{~Bq} \mathrm{~m}^{-3}$, whereas the total activity is similar. This caesium-134 inversion is displayed in Fig. 13, where the relative humidity scheme has been used.

\subsection{Errors diagnosis}

The inversion offers a diagnosis of the errors. Indeed $\boldsymbol{\mu}=\boldsymbol{H} \overline{\boldsymbol{\sigma}}+\overline{\boldsymbol{\varepsilon}}$, and the measurements are diagnosed and corrected to be $\boldsymbol{\mu}-\overline{\boldsymbol{\varepsilon}}$. A scatterplot is given in Fig. 14 to compare $\boldsymbol{\mu}-\overline{\boldsymbol{\varepsilon}}$ to $\boldsymbol{\mu}$. A too fine agreement between the two would indicate the inversion acts as a mere fitting, which would make the estimation unreliable, given the current flaws of dispersion model and other sources of errors. On the contrary, the scatterdiagram has a similar aspect to the scatterdiagram of the observations versus simulated observations (through the model and the UNSCEAR report source estimation). This indicates the inversion is roughly able to diagnose the errors in the observations and/or the model. This qualitative error diagnosis was very helpful to understand that an arbitrary choice of the tuning parameters $(m, \chi)$ is inadequate.

\section{Conclusions}

In this paper, a reconstruction of the source of the farreaching radionuclides from the Chernobyl accident was performed. The retrieval is based on advanced inverse modelling techniques which, in particular, exploit the positivity of the source. The main work was carried out on ${ }^{131} \mathrm{I}$, and ${ }^{137} \mathrm{Cs}$ and ${ }^{134} \mathrm{Cs}$ to a lesser extent. A typical inversion has been exhibited for ${ }^{131}$ I. Its main characteristics were identified using a sensitivity analysis to the parameters of the inversions. The two parameters to which the reconstructions are mainly sensitive are the mandatory prior mass scale $m$ and the prior error variance $\chi$. In all configurations (species, wet scavenging), they have been estimated using the L-curve technique (based on a posteriori analysis of the inversions). The total iodine-131 retrieved released activity lies essentially in the range $1.5 \times 10^{18}-2.0 \times 10^{18} \mathrm{~Bq}$ which is compatible with 

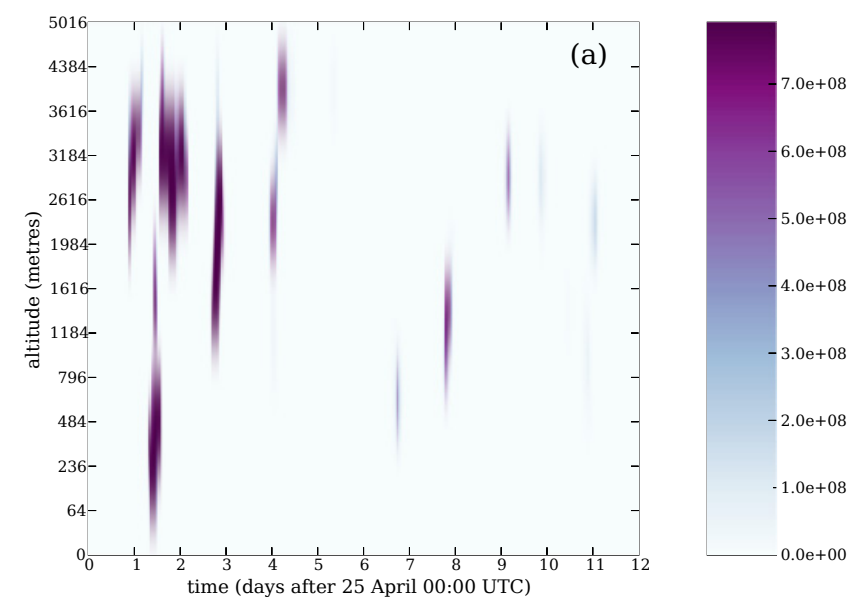

Fig. 12. The same as for Fig. 3, but for caesium-137.
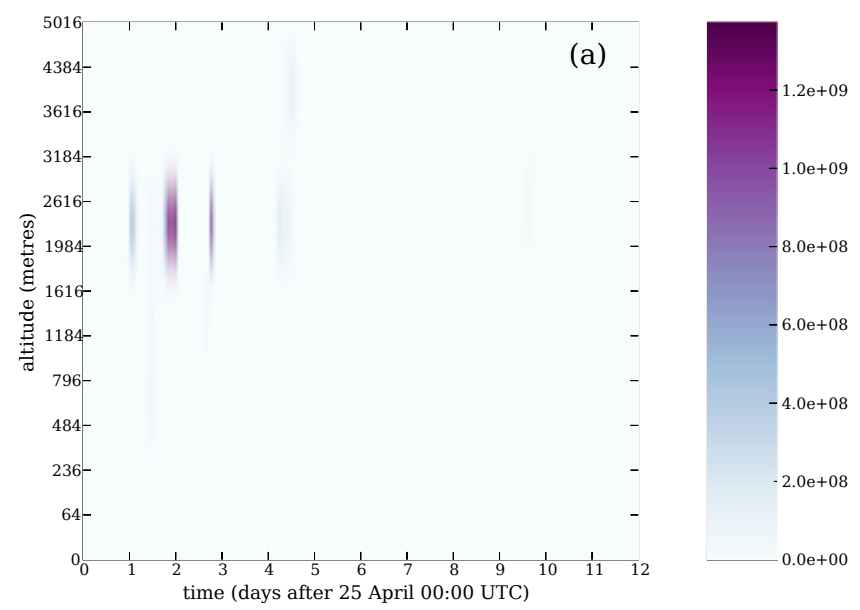

Fig. 13. The same as for Fig. 3, but for caesium-134.

the latest estimation of the Chernobyl radionuclides emission. The inversion is two-dimensional, time and altitude, so that fine details of the chronology are probed. The threestage scenario is recovered in all inversions. In the first stage, surges are identified as many secondary sources spread from the ground to the top of the inversion grid. The retrieved spot connected to the initial explosion is substantial above $800 \mathrm{~m}$, whereas the second spot matches chronologically the ignition of the combustible gas. The third stage exhibits several spots from the ground up to $3500 \mathrm{~m}$. It was stressed that the details of the third stage are not strongly constrained, essentially by measurements from Greece. It was also explained that the reconstructed temporal details are likely to be more reliable than the vertical details, because of the sensitivity of measurements to such details.

Inversion were also obtained for ${ }^{137} \mathrm{Cs}$ and ${ }^{134} \mathrm{Cs}$, with a total released mass of $13.6 \times 10^{16} \mathrm{~Bq}$ and $3.5 \times 10^{16} \mathrm{~Bq}$, re-
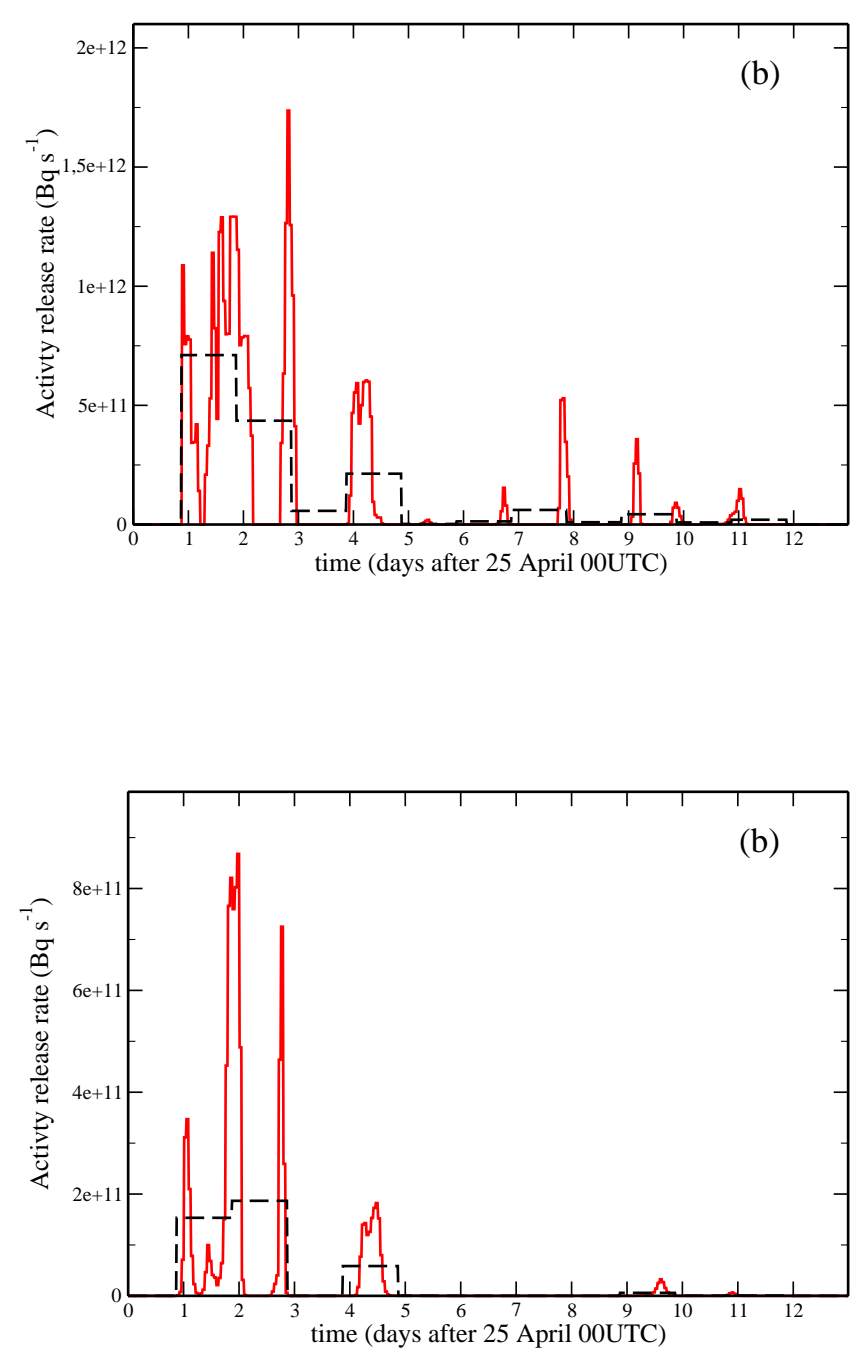

spectively, of the same order as the consensus estimations. From the estimation of $(m, \chi)$ has been learnt how much peaked the retrieved sources are, but also how reliable the observations are in the view of the inverse modelling system.

There are several ways to improve these reconstructions that we would like to mention. One consists in the refinement of the present work (higher space resolution in the adjoint solutions computations, improvements of the physical parametrisations). A promising one would be to use earlier estimations as a prior. The inversions performed in this paper only used a scale $m$ that loosely depend on earlier estimation. It was mandatory not to use the earlier temporal profile as a background, since the inversion results were meant to be as independent as possible from previous reconstruction works. This being done, an inversion with a first guess constructed from earlier estimations could be performed and interpreted as a refinement of earlier estimations from inverse modelling 


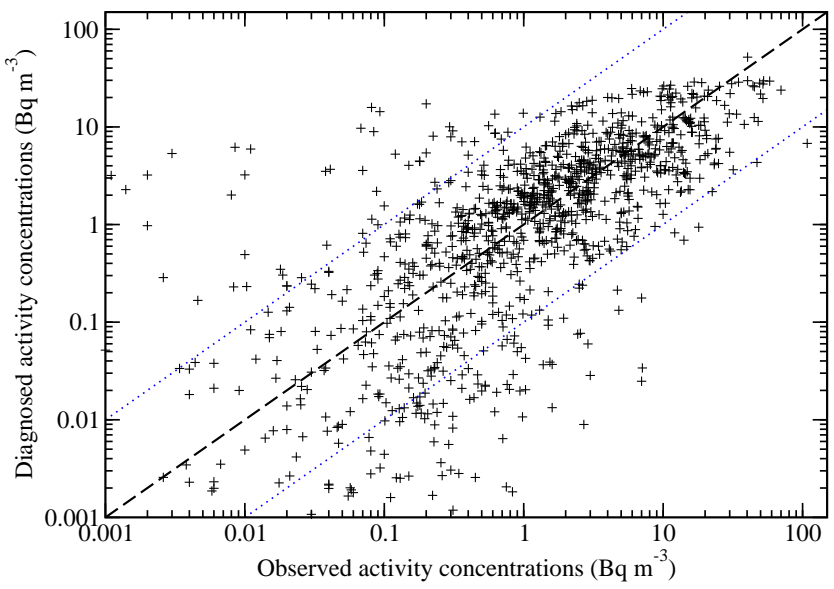

Fig. 14. Scatterdiagram of the diagnosed concentrations (which identify with $\boldsymbol{\mu}-\overline{\boldsymbol{\varepsilon}}$ ) versus the measurements.

of observations. This could provide with a compromise between reconstructions of different nature (dispersion analysis from meteorological data, inverse modelling, core inventories) and using different kind of observations (concentrations, deposition).

One could also improve physical parametrisations, though keeping simple and linear parametrisations, so that the inverse modelling remains simple to perform. As suggested to us by J. Pudykiewicz, this could start by a better diagnostic of the wet scavenging coefficient $\Lambda^{s}$ of the relative humidity parametrisation (wet scavenging being the most efficient removal process for the Chernobyl dispersion).

And yet, the most direct way to improve the inverse modelling approach would be to use activity concentrations sampled in Ukraine, Belarus, Poland, etc, but these are not directly available.

Acknowledgements. The authors would like to thank D. Quélo and M. Krysta for sharing their expertise, Y. Minier and M. De Cort for their help with the REM database. They also thank J. Pudykiewicz for his review and his suggestions. M. Bocquet thanks M. Sofiev for a useful discussion.

Edited by: S. Galmarini

\section{References}

Albergel A., Martin, D., Strauss, B., and Gros, J. M.: The Chernobyl accident: modelling of dispersion over Europe of the radioactive plume and comparison with activity measurements, Atmos. Environ., 22, 2431-2444, 1988.

Baklanov, B. and Sørensen, J. H.: Parameterisation of radionuclides deposition in atmospheric long-range transport modelling, Phys. Chem. Earth (B), 26(10), 787-799, 2001.
Baklanov, A.: Parameterisation of the deposition processes and radioactive decay: a review and some preliminary results with the DERMA model, DMI, 99-4, 1999.

Bocquet, M.: Grid resolution dependence in the reconstruction of an atmospheric tracer source, Nonlin. Processes Geophys., 12, 219-234, 2005,

http://www.nonlin-processes-geophys.net/12/219/2005/.

Bocquet, M.: Reconstruction of an atmospheric tracer source using the principle of maximum entropy. I: Theory, Quart. J. Roy. Meteorol. Soc., 131, 2191-2208, 2005.

Bocquet, M.: Reconstruction of an atmospheric tracer source using the principle of maximum entropy. II: Applications, Quart. J. Roy. Meteorol. Soc., 131, 2209-2224, 2005.

Bocquet, M.: High resolution reconstruction of a tracer dispersion event: application to ETEX, in press, Quart. J. Roy. Meteorol. Soc., 2007.

Boutahar, J., Lacour, S., Mallet, V., Quélo, D., Roustan, Y., and Sportisse, B.: Development and validation of a fully modular platform for numerical modelling of air pollution: Polair3D, Int. J. Environ. and Pollution, 22, 1-2, 2004.

Brandt, J.: Modelling Transport, Dispersion and Deposition of Passive Tracers from Accidental Releases, PhD thesis, NERI, Denmark, 1998.

Brandt, J., Christensen, J. H., and Frohn, M.: Modelling transport and deposition of caesium and iodine from Chernobyl accident using the DREAM model, Atmos. Chem. Phys., 2, 397-417, 2002 ,

http://www.atmos-chem-phys.net/2/397/2002/.

De Cort, M., Dubois, G., Fridman, S. D., Germenchuk, M., G., Izrael, Y. A., Janssens, A., Jones, A. R., Kelly, G., N., Kvasnikova, E., V., Matveenko, I., I., Nazarov, I., N., Pokumeiko, Y., M., Sitak, V. A., Stukin, E., D., Tabachny, L., Y., Tsaturov, Y. S., and Avdyushin, S., I.: Atlas of caesium deposition on Europe after the Chernobyl accident, Luxembourg, Office for Official Publications of the European Communauties, ISBN 92-828-3140-X, 1998.

Devell, L.,Güntay, S., and Powers, D. A.: The Chernobyl Reactor Accident Source Term: Development of a Consensus View. Paris, CSNI Report, OECD/NEA, 1995.

Gudiksen, P. H., Harvey, T. F., and Lange, R.: Chernobyl Source Term, Atmospheric Dispersion, and Dose Estimation, Health Physics, 57(5), 697-706, 1989.

Hansen, C.: Analysis of discrete ill-posed problems by means of the L-curve, SIAM Review, 34, 561-580, 1992.

Hass, H., Memmesheimer, H., Geiss, H., Jakobs, H., J., Laube, M., and Ebel, A.: Simulation of the Chernobyl Radioactive Cloud over Europe using the EURAD model, Atmos. Environ., 24A, 676-692, 1990.

Issartel, J.-P. and Baverel, J.: Inverse transport for the verification of the Comprehensive Nuclear Test Ban Treaty, Atmos. Chem. Phys., 3, 475-486, 2003, http://www.atmos-chem-phys.net/3/475/2003/.

Jaynes, E. T.: Information statistics and statistical mechanics, Phys. Rev., 106, 620, 1957.

Jaynes, E. T.: Information statistics and statistical mechanics II. Phys. Rev., 108, 171, 1957.

Krysta, M. and Bocquet, M.: Source reconstruction of an accidental radionuclide release at European scale, Quart. J. Roy. Meteorol. Soc., in press, 2007. 
Krysta, M.: Modélisation numérique et assimilation de données de la dispersion de radionucléides en champ proche et à l'échelle continentale, PhD thesis, Paris XII university, 2006.

Hourdin, F. and Talagrand, O.: Eulerian backtracking of atmospheric tracers. I: Adjoint derivation and parametrization of subgid-scale transport, Quart. J. Roy. Meteorol. Soc., 132, 585603, 2006.

Lange, R., Dickerson, M. H., and Gudiksen, P. H.: Dose estimates from the Chernobyl accident. Nucl. Technol., 82, 311-322, 1988.

Louis, J. F.: A parametric model of vertical eddy fluxes in the atmosphere, Boundary-Layer Meteor., 17, 197-202, 1979.

Persson, C., Rodhe, H., and De Geer, L.-E.: The Chernobyl accident - A meteorological analysis of how radionuclides reached and were deposited in Sweden, Ambio, 16, 20-31, 1987.

Pudykiewicz, J. A.: Simulation of the Chernobyl dispersion with a 3-D hemispheric tracer model, Tellus, 41B, 391-412, 1989.

Pudykiewicz, J. A.: Application of adjoint transport tracer equations for evaluating source parameters, Atmos. Environ., 32, 3039-3050, 1998.

Quélo, D., Krysta, M., Bocquet, M., Isnard, O., Minier, Y., and Sportisse, B.: Validation of the POLYPHEMUS platform: the ETEX, Chernobyl and Algeciras cases, Atmos. Environ., in press, 2007.
Rietsch, E.: The maximum entropy approach to inverse problems: spectral analysis of short data records and density structure of the Earth, J. Geophys., 42, 489-506, 1977.

Talerko, N.: Reconstruction of ${ }^{131}$ I radioactive contamination in Ukraine caused by the Chernobyl accident using atmospheric transport modelling, J. Environ. Radioactivity, 84, 343-362, 2005.

Uliasz, M.: Application of the Perturbation Theory to the Sensitivity Analysis of an Air Pollution Model, Z. Meteor., 33, 6, 355362, 1983.

United Nations: Sources and effects of ionizing radiation. United Nations Scientific Commitee on the Effects of Atomic Radiation. Report to General Assembly, United Nations, New-York, 2000.

Verwer, J. G., Hundsdorfer, W. H., and Blom, J. G.: Numerical Time Integration for Air Pollution Models, Proceedings of the Conference APMS'98, 26-29 October, ENPC-INRIA, 1998.

Waight, P., Metivier, H., Jacob, P., Soulchkevitch, G., Viktorsson, C., Bennett, B., Hance, R., Yumazawa, S., Kusumi, S, Bouville, A., Sinnaeve, J., Ilari, O., and Lazo, E.: Chernobyl Ten Years On. Radiological and Helth Impact, an Asseessment by the NEA Committee on the Radiation Protection and Public Health, OECD Nuclear Agency, 1995. 